\title{
Enlargement of salivary glands in bulimia
}

\author{
Josef Vavrina, M.D., Werner Müller, M.D., Jan-Olaf Gebbers, M.D.*
}

\begin{abstract}
We report an unusual case of bulimia nervosa with bilateral swelling of parotid and submandibular glands as the only symptom of the underlying behavioural disorder. Histologically, sialadenosis was diagnosed in a parotid biopsy. The parotomegaly in bulimia may be a diagnostic primer as these patients often deny their eating disorder. B-scan ultrasonography is an important diagnostic tool to assess the nature of the parotid enlargement. Hyperamylasaemia occurs commonly in bulimic patients and may help to confirm the diagnosis. All patients with suspected bulimia should have a thorough medical history and physical examination to rule out other aetiologies of asymptomatic parotid swelling. As the enlargement is usually transient surgical intervention is only rarely required.
\end{abstract}

Key words: Amylases; Bulimia; Salivary gland diseases

\section{Introduction}

Bulimia nervosa is an eating disorder characterized by binge eating coupled with self-induced vomiting. Laxative and diuretic abuse is a feature in some cases. This disorder typically occurs in late adolescence or early adulthood, primarily in women with only five to 10 per cent of the cases being men (Pyle et al., 1986). In contrast to the emaciated appearance of patients with anorexia nervosa, patients with bulimia have few, if any, observable signs of the behavioural disorder. Hypertrophy of the salivary glands as well as dental enamel erosions may be presenting symptoms that can be used clinically to aid in the diagnosis (Taylor and Sneddon, 1987). On the other hand bulimic patients usually hide their eating behaviour so that the psychosomatic origin of the enlarged salivary glands may remain obscure and extensive diagnostic steps are made. In otolaryngological literature these leading signs of bulimia have received poor attention.

\section{Case report}

A 24-year-old athletic married man, father of one child, was referred to the ENT clinic because of a two-month history of progressive bilateral painless parotid swelling and general weakness. He had no history compatible with Sjögren's syndrome, sarcoidosis, liver disease or other diseases associated with parotid swelling. He had received no medications known to cause parotid swelling. He weighed $64 \mathrm{~kg}$ and was $1.8 \mathrm{~m}$ tall. The soft enlarged parotids (Figure 1) were not tender and were associated with submandibular enlargement. There were no palpable lymph nodes, no enamel erosions and the remainder of the physical examination was normal.

Laboratory abnormalities included an elevated serum amylase value of $132 \mathrm{U} . / /$ (normal <65 U./l) with no clinical evidence of pancreatic disease. Results of electrolyte profile, erythrocyte sedimentation rate, differentiated blood count, glucose, glucose tolerance test, serum $\mathrm{pH}$, albumin, serum electrophoresis, antinuclear antibodies, immunoglobulins and serology were all within normal limits. Chest roentgenogram was normal; electrocardiogram showed a sinus bradycardia. B-scan sonography revealed extremely enlarged homogenous parotid and submandibular glands with neither signs of acute or chronic inflam- mation nor neoplastic changes. CT scan of head, neck, chest and abdomen confirmed hyperplasia of all salivary glands but showed no other abnormalities. Histologically, an enlargement of the serous acinar cells with a slight compression of the ducts by the swollen acini was found in the biopsy of the parotid gland (Figure 2 ). The densely packed mature enzyme granules in the cytoplasm of the acinar cells have displaced the nuclei towards the basal part of the cells. Inflammatory changes were absent. The findings are consistent with sialadenosis of the granular type (Seifert, 1991).

Further inquiries into his past history made the case clearer. The patient's wife reported that her husband had had phases of voracious appetite with intermittent vomiting for nine months. Psychiatric opinion was that our patient satisfied the DSM IV diagnostic criteria for bulimia nervosa with secondary depression. He was referred to a psychotherapist. When seen as an outpatient after six months, although bulimia and vomiting persisted, the enlargement has diminished but had not entirely disappeared.

\section{Discussion}

Eating disorders seem to have become more common, especially among young women (Hoek, 1991). A survey of 355 college students determined the prevalence of the binge-eating-syndrome as 13 per cent within the normal college population (Halmi et al., 1981). However, our case shows that even in married men bulimia should be considered. Up to 50 per cent of patients with bulimia will develop intermittent or persistent parotomegaly (Mitchell et al., 1985). In some cases of bulimia the facial swelling is the only notable feature (Taylor and Sneddon, 1987). The parotomegaly is always bilateral, diffuse and painless. It is often recurrent and lacks any signs of inflammation (Levin et al., 1980; Ogren et al., 1987). Enamel erosions are another typical sign in about one-third of patients with bulimia due to the irritative effect of the gastric acid (Rauch and Herzog, 1987; Mandel and Kaynar, 1992). Some patients may also show callouses in the knuckle region due to insertion of the fingers into the mouth to induce vomiting (Mandel and Kaynar, 1992).

As discussed by Seifert (1991), sialadenosis is a noninflam- 


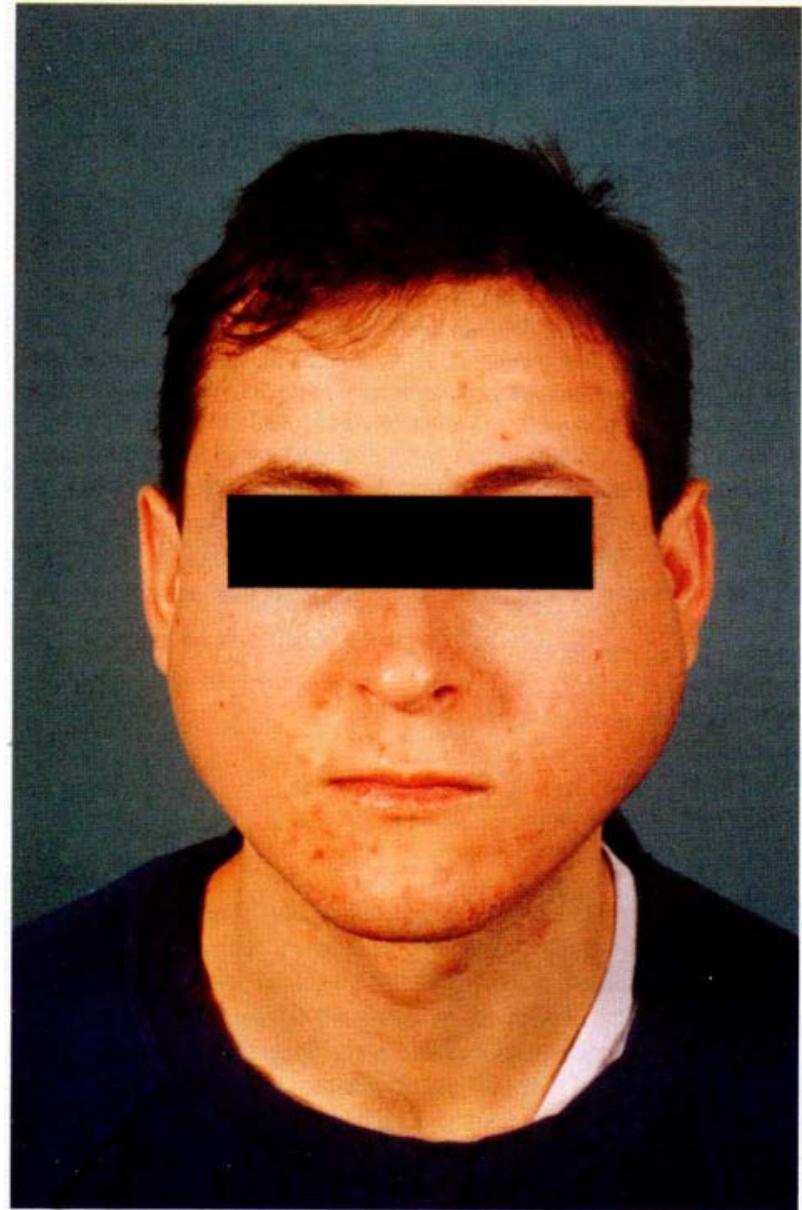

Fig. 1

Bilateral enlargement of parotid glands in a patient with bulimia nervosa.

matory salivary gland disorder accompanied by metabolic and secretory changes of the gland parenchyma and by recurrent painless bilateral swelling, usually of the parotid glands. The lesion has been related to nutritional, endocrine and metabolic disorders (Katsilambros, 1961; Davidson et al., 1969). The pathogenesis of this enlargement is not clear. Also in bulimia the exact mechanism causing the enlargement of salivary glands is unknown. Dreiling et al (1978) supposed that the parotids hypertrophy in response to trophic stimuli released by the pancreas responding to stimuli. Recent studies suggest that the development of sialadenosis in bulimia is related to enzymatic secretory disturbances due to autonomic nerve dysfunction (Riad et al., 1991).

The diagnosis of bulimia nervosa is made on clinical grounds. No specific diagnostic tests exist (Foster, 1992). All patients with suspected bulimia should have a thorough medical history and physical examination to rule out other causes of asymptomatic parotid swelling such as Sjögren's disease, sarcoidosis or gastrointestinal diseases (Gill, 1977). An electrocardiogram is desirable for most patients (Mitchell et al., 1987). In bulimia the blood profile may show low potassium, especially if laxatives have been taken, and a high bicarbonate level indicates recurrent vomiting. Hyperamylasaemia, caused by increased salivarytype amylase activity, without clinical evidence of pancreatitis could be a useful clinical marker in patients with suspected bulimia (Humphries et al., 1987). Ultrasonography is a noninvasive low cost diagnostic tool used to rule out masseteric hypertrophy, sialolithiasis, sialadenitis or neoplasm.

Bulimia nervosa is a chronic illness which requires a longterm psychotherapy. Recently, Mehler and Wallace (1993) reported a successful treatment of sialadenosis in two bulimic

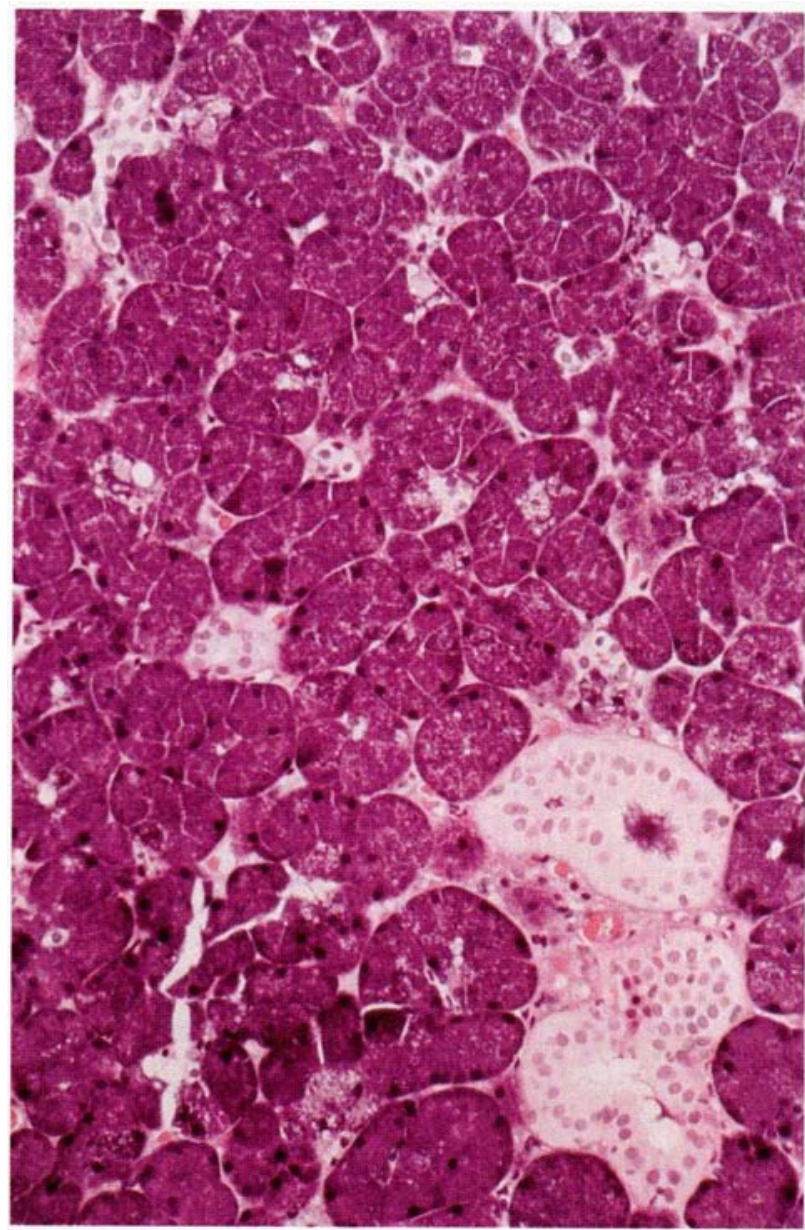

FIG. 2

Light microscopy of the parotid gland biopsy: enlarged acinar cells stuffed with enzyme granules and displaced nuclei, slight compression of ducts by the swollen acini. These findings are consistent with sialadenosis of the granular type. (H \& E; medium magnification).

patients with pilocarpine. Berke and Calcaterra, (1985) proposed superficial parotidectomy in managing the cosmetic and psychological aspects of these patients. In contrast, Rauch and Herzog (1987) believe that surgical management is contraindicated because of the surgical risks, the natural history of the disorder and the patient's psychological state. In addition, it seems that some patients with eating disorders are not true cases of bulimia nervosa but have depression. When treated with antidepressants vomiting and parotid enlargement may disappear (Lavender, 1969). Furthermore, after treatment of their bulimia salivary gland enlargement resolves in most cases (Ogren et al., 1987). When salivary gland hypertrophy persists, it must be suspected that the patient is still engaging in binge-eating behaviour. Also in our patient the enlargement of the salivary glands seems to be transient in nature.

\section{References}

Berke, G. S., Calcaterra, T. C. (1985) Parotid hypertrophy with bulimia: a report of surgical management. Laryngoscope 95: 597-598.

Davidson, D., Leibel, B. S., Berris, B. (1969) Asymptomatic parotid gland enlargement in diabetes mellitus. Annals of Internal Medicine 70: $31-38$.

Dreiling, D. A., Nuronna, M., Nacchieko, M., Peironi, P., Wolfson, P. (1978) The parotid and pancreas. American Journal of Gastroenterology 70: 627-634.

Foster, D. W. (1992) Anorexia nervosa and bulimia. In Harrison's Principles of Internal Medicine. 12th Edition. (Wilson, J. D., 
Braunwald, E., Isselbacher, K. J., Petersdorf, R. G., Martin, J. B., Fauci, A. S., Root, R. K., eds.), McGraw-Hill Book Co., New York, pp 417-420.

Gill, G. (1977) Metabolic and endocrine influences on the salivary glands. Otolaryngologic Clinics of North America 10: 363-369.

Halmi, K. A., Falk, J. R., Schwarts, E. (1981) Binge eating and vomiting: a survey of college populations. Psychological Medicine 11: 697-706.

Hoek, H. W. (1991) The incidence and prevalence of anorexia nervosa and bulimia nervosa in primary care. Psychological Medicine 21: $455-460$.

Humphries, L. L., Adams, L. J., Eckfeldt, J. H., Levitt, M. D., McClain, C. J. (1987) Hyperamylasemia in patients with eating disorders. Annals of Internal Medicine 106: 50-52.

Katsilambros, L. (1961) Asymptomatic enlargement of the parotid glands. Journal of the American Medical Association 178: 173-174.

Lavender, S. (1969) Vomiting and parotid enlargement. Lancet 1: 426 (Letter).

Levin, P. A., Falko, J. M., Dixon, K., Gallup, E. M., Saunders, W. (1980) Benign parotid enlargement in bulimia. Annals of Internal Medicine 93: 827-829.

Mandel, L., Kaynar, A. (1992) Bulimia and parotid swelling. Journal of Oral and Maxillofacial Surgery 50: 1122-1125.

Mehler, P. S., Wallace, J. A. (1993) Sialadenosis in bulimia. A new treatment. Archives of Otolaryngology, Head and Neck Surgery 119: $787-788$.

Mitchell, J. E., Hatsukami, D., Eckert, E. D., Pyle, R. L. (1985) Characteristics of 275 patients with bulimia. American Journal of Psychiatry 142: 482-485.
Mitchell, J. E., Seim, H. C., Colon, E., Pomeroy, C. (1987) Medical complications and medical management of bulimia. Annals of Internal Medicine 107: 71-77.

Ogren, F. P., Huerter, J. V., Pearson, P. H., Antonson, C. W., Moore, G. F. (1987) Transient salivary gland hypertrophy in bulimics. Laryngoscope 97: 951-953.

Pyle, R. L., Halvorson, P. A., Neumann, P. A., Mitchell, J. E. (1986) The increasing prevalence of bulimia in freshman college students. International Journal of Eating Disorders 5: 631-647.

Rauch, S., Herzog, D. (1987) Parotidectomy for bulimia: a dissenting view. American Journal of Otolaryngology 8: 376-380.

Riad, M., Barton, J. R., Wilson, J. A., Freeman, C. P. L., Maran, A G. D. (1991) Parotid salivary secretory pattern in bulimia nervosa. Acta Otolaryngologica (Stockholm) 111: 392-395.

Seifert, G. (1991) Histological typing of salivary gland tumours. In WHO International Histological Classification of Tumours. Springer, Berlin/Heidelberg/New York, p 34.

Taylor, V. E., Sneddon, J. (1987) Bilateral facial swelling in bulimia. British Dental Journal 163: 115-117.

Address for correspondence:

Dr Josef Vavrina,

ENT Department,

Kantonsspital,

6000 Luzern 16

Switzerland.

Fax: (0041) 41254995 